\title{
On Pedagogical Strategies to Develop Undergraduates' Chinese-English Pragmatic Translation Competence
}

\author{
Jun Chen ${ }^{1, a}$, Yuhong Huang ${ }^{2, b}$
}

${ }^{1}$ College of Humanities and Law, Fujian Agriculture and Forestry University, Fuzhou, China

${ }^{2}$ College of Humanities and Law, Fujian Agriculture and Forestry University, Fuzhou, China

acjgullit@163.com, b47098368 @qq.com

Keywords: Pragmatic translation competence development, PACTE model, Pedagogical strategies.

\begin{abstract}
Based on PACTE's translation competence model, this paper presents the five components of $\mathrm{C}$-E pragmatic translation competence. It argues that bilingual communication sub-competence is the kernel of Chinese-English pragmatic translation, while translation knowledge sub-competence, strategic sub-competence, extra-linguistic sub-competence and instrumental sub-competence guarantee the functioning of bilingual communication sub-competence. It also proposes five pedagogical strategies to develop undergraduates' C-E pragmatic translation competence by conducting a case study of teaching pragmatic translation course.
\end{abstract}

\section{本科生汉英应用翻译能力的培养}

\author{
陈隽 $1, a ，$ 黄玉虹 ${ }^{2, b}$ \\ 1 福建农林大学文法学院, 福州, 福建, 中国 \\ 2 福建农林大学文法学院, 福州, 福建, 中国 \\ acjgullit@163.com, b47098368@qq.com
}

关键词：应用翻译能力培养; PACTE翻译能力模型; 教学策略

中文摘要. 基于PACTE小组提出的翻译能力模型, 分析本科生汉英应用翻译能力的构成, 提 出本科学习者的汉英应用翻译能力应以双语交际能力为核心, 辅以翻译专业知识、策略能力、 语言外能力和工具能力。同时, 根据应用翻译课程的教学实际, 探讨发展本科生汉英应用翻 译五项能力的教学策略和方法。

\section{1. 引言}

当前, 中国与国际社会的融合进一步加深。要在国际上唱响中国声音, 讲好中国故事, 中国文化要 “走出去” , 少不了语言服务业这一助推器和润滑剂。有报告指出, “截至2013 年底, 有 $64 \%$ 的翻译服务企业中译外业务量占业务总量的一半以上，显著高于外译中”，“翻 译服务的业务量集中体现在金融业、制造业、信息技术产业、电力、热力、燃气” 等非文学 翻译领域。[1]在这一背景下, 研究汉英应用翻译能力的构成与发展就具有迫切的现实意义。

\section{2. 翻译能力研究现状综述}

随着市场经济对职业翻译的需求激增, 翻译能力研究近年来成为学界研究的热点。21世 纪以降, 国内外关于翻译能力的主要研究可归类如下: 
（1）关注翻译能力的本质、构成以及习得过程 (Neubert, 2000; PACTE, 2000, 2003, 2005, 2008, 2009, 2011; Chesterman, 2000; 杨晓荣, 2002; 姜秋霞、权晓辉, 2002; 刘宓庆, 2003; 苗菊，2007；王树槐，2008；杨志红，2014；傅敬民，2015）

（2）基于发展翻译能力的人才培养和翻译教学研究 (文军，2004; 高华丽，2008; Colina, 2009; 王金波, 2009; 全亚辉, 2010; 李瑞林, 2011; 刘和平, 2011; Schaffner, 2012; 金萍, 2012; 王树槐，2013; 方红，2014；吴望，2015; 祝朝伟，2015）

（3）基于翻译能力的翻译测试研究（穆雷，2006; 罗选民，2008; Angelelli, 2009; 王克 非、杨志红, 2010; 陈怡, 2010; 肖维青, 2012)

（4）关注译出翻译 (汉译英) 能力的培养 (刘士聪，2007; 杨晓荣，2008; 王宏，2012; 马会娟, 2010，2012，2013; 韩孟奇，2015）

可以看出, 翻译能力研究已经取得了一定的成果, 既有对翻译能力构成模式的关注, 也 将研究成果应用于教学的主要环节。但是, 大多数的研究是以从外语翻译到母语为落脚点, 而对于从母语翻译到外语的研究, 特别是汉英应用翻译能力的关注较少, 而这也正是本文的 研究目的所在。

\section{3. 汉英应用翻译能力的定义、构成及解读}

从现有研究来看, 学者们对翻译能力的定义和构成并未达成一致的共识。参考马会娟对 翻译能力的定义 ${ }^{[2]}$, 可以将汉英应用翻译能力定义为译者能够胜任汉英应用翻译任务所必备 的潜在知识和技能体系。

王树槐按照翻译能力性质与构成 ${ }^{[3]}$, 将中西方的翻译能力模式划分为 11 类: 天赋说、自 然展开说、自然展开修正说、建构说、转换说、策略或认知说、语篇协调说、生产一选择说、 交际说、语言一语篇能力说、多因素均力说。马会娟在西班牙PACTE小组 (Process in the Acquisition of Translation Competence and Evaluation, 翻译能力习得过程和评估专项研究小组) 构建模式的基础上, 结合翻译的教学实际, 提出汉译英翻译能力由五个成分构成 ${ }^{[2]}$ :

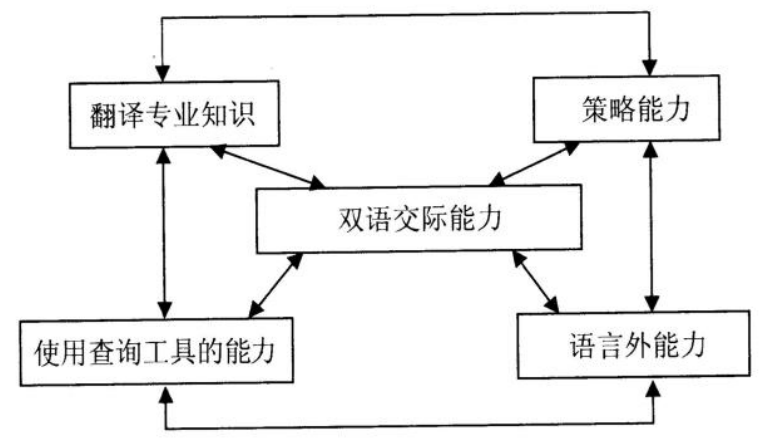

图1: 汉译英能力模式

上述模式可解读如下：双语交际能力体现为 “译者在双语转换过程中所激活并使用的词 汇、语法、语篇、语用和社会语言等知识” [4]。笔者曾对福建农林大学英语专业三年级的学 生进行过问卷调查, $95 \%$ 以上的同学都反映汉译英过程中, 译者面临的主要困难在于用目的 语进行通顺、流畅、自如地表达。翻译质量的高低与译者的译语语言水平成正比关系, 双语 交际能力是汉译英过程中的核心能力。

翻译专业知识包括指导翻译实践、方法的理论知识和翻译职业常识和翻译伦理。策略能 力指的是译者在进行语言转换时找到解决难题方法的能力。语言外能力 “主要是陈述性知识, 包括对世界的普遍认知和双文化知识、百科知识等” [5]。工具能力包括译者在翻译过程中查 询信息的能力。这四种能力紧密联系, 互联互动, 为译者充分发挥双语交际能力提供保障。 


\section{4. 发展本科生汉英应用翻译能力的教学策略}

\section{1 练讲并重, 打磨双语交际能力}

翻译教学旨在 “培养、造就并发展学习者在语际交流重的语言交际能力。” ${ }^{[6]}$ 语言能力 的提升非一朝一夕之功。只有在输入量达到一定的程度时, 才会引发语言输出的质变。从这 一层面上说, 应用翻译课程是建立在精读、泛读等学科基础课程之上的。换言之, “要从培 养英语理解能力、阅读能力、写作能力入手，辅之以汉英翻译入门技能的训练” [7]，在大量 接触原汁原味、各种体裁英语原文和保证一定训练量的基础上, 发展学生的汉英翻译能力。 另一方面, 在进行翻译练习时, 应该鼓励学生分组讨论, 分享译文, 通过生一生之间的译文 互赏互评、取长补短, 促使学生共同提高语言能力。请看下例:

例1: 我校举办2013-2014学年大学生心理沙龙活动

译文1：Students' Psychological Salon of 2013-2014 Is Held in FAFU

译文2: FAFU held students' psychological salon during the period of 2013 and 2014

译文3: Our University Hold the Students' Psychological Salon

译文4: FAFU Holds Psychological Salon Activities

在进行翻译练习之前, 布置学生进入China Daily的网页，阅读并总结英语新闻标题的共 性。在比较讨论的基础上, 学生认为, 译文1没有注意到英语新闻标题常省略be动词这一特征; 译文2不够简洁, 且英语新闻标题一般多采用现在时, 以拉近新闻事件与读者之间的距离; 译 文3没有注意到our university一词在不同读者看来含义不同，容易产生误解，不符合新闻英语 客观明晰的特征, 而译文4是正确的翻译。

通过类似的分组讨论，学生意识到应用翻译是要考虑目的语表达习惯和译文预期功能的 活动, 而搬字过纸的字字对应式翻译离达到双语交际的目标还有很大距离。在意见不统一时, 学生通过查阅资料, 相互切磋, 在练习和研讨并重的过程中逐步积累、提高语言交际能力。

\section{2 理论讲解, 传授翻译专业知识}

翻译专业知识对于提高翻译能力是不可或缺的一环。然而, 理论对于翻译实践是否有用 一直以来都备受争议。事实上, “对于以提高翻译能力为目标的笔译教学来说, 教师没有必 要传授纯理论, 应用理论中的翻译专业知识才是教学的重点” [8]。在实际教学中, 教师应该 注意理论与实践的结合度, 把翻译专业知识传授与翻译训练结合起来, 在讲评练习、点拨疑 难时, 有意识地从专业知识层面给予解答, 引导学生把握具有普遍性的翻译规律, 并能灵活 运用于翻译实践活动。例如:

例2: 爱心专座 (公交车上的告示牌)

译文1: Love Seats

译文2: Seats Reserved for the Old, the Weak, the Sick, the Disabled and the Pregnant

在讲解公示语翻译时, 笔者举了上述的例子, 并让学生评论上述两个译文质量。学生指 出: 译文 1 为误译, love seat在英文里指的是 “情侣座, 鹗冨座”; 译文2把原文的内涵直译出 来, 译文不仅穴长, “老弱病残” 的直译也容易让人心生不快。教师此时可以因势利导地引 入纽马克提出的 “交际翻译” 理论: 交际翻译关注受众, 译者要排除阅读或交际上的障碍, 为读者创造交际顺利进行的环境, 强调 “译文在译文读者身上取得的效果要和原文在原文读 者身上取得的效果近似” [9]，而上述译文明显没能做到。通过以上翻译专业知识的解读，学 生能够在实例训练中掌握到交际翻译理论的核心, 今后在碰到类似翻译时也会有意识地思考 译文是否达到了既定的交际效果，达到理论与实践结合的效果。

\section{3 读译结合, 培养翻译策略能力}

应用文本的翻译要达到预期的目的和功能, 关键是译者要对目的语中相关文本的规约和 习惯表述方式有所了解。换言之, 一则不符合目标读者阅读习惯的译文, 即便用词准确, 语 言优美，恐怕也达不到预期的效果。因此，应用翻译的教学过程中，要灌输 “看易写” 和 “模 
仿一借用一创新” 的翻译策略 ${ }^{[10]}$ 。具体来说, 可以引导学生在翻译之前, 先大量地接触、阅 读英语世界中某一题材的真实语料, 熟悉目的语文本的行文风格和表述习惯。翻译过程中, 对英语中现有的对应表达可以采取 “拿来主义”，对英语中的类似表达可以改造利用，对中 国特色的表达则要按照英语的习惯和思路进行创译和解释说明。例如:

例3：我校第六届大学生职业生涯规划决赛邀请了职业指导专家 $X X$ 、省十佳职业生涯规 划之星 $X X X$ 、省农村信用联社人力资源管理师 $X X X$ 、公司企划高管 $X X X$ 、国家职业生涯规划师 $X X X$ 和……有限公司销售一区总监 $X X X$ 担任评委。

从学生上交的练习发现, 大多数学生对于原文中具有中国特色的人名和官职头衔（斜体 部分）采取了直译的处理方式。一些同学对职称头衔和公司名没有经过查询, 使得译文的准 确性不高。教师在评讲时可以突出这方面的问题, 引导学生回到英语新闻中去寻求解决策略: 读者的注意力不会放在不熟悉的人名和官职头衔上, 因此直译后圥长的文本可能会造成阅读 负担。正因为如此, 英语新闻中对不为人知的人名往往采用省译的处理策略, 对官职头衔则 可以分类翻译。故此，原文中的 “职业指导专家”、“省十佳职业生涯规划之星” 和 “国家 职业生涯规划师” 可以归类为career planning and development experts， “省农村信用联社人力 资源管理师” 可译为human resources professional，而 “公司企划高管” 和 “......有限公司销售 一区总监”则可以合译为senior management executives.

通过这样的比读和评讲, 引导学生掌握处理汉英应用翻译中的难点, 并且可以再设计针 对性的翻译练习，使学生习得的翻译策略能力得以巩固。

\section{4 巧选文本, 扩大语言外知识}

普遍认为, 语言外知识包括主题知识、百科知识和文化能力三部分。应用翻译的范围涵 盖社会生产生活的方方面面, 对译者的知识面是一大考验。教师在课堂练习时, 要尽可能选 择 “与职业翻译的实际相接近的，各种文体、领域的真实材料” [11]。例如:

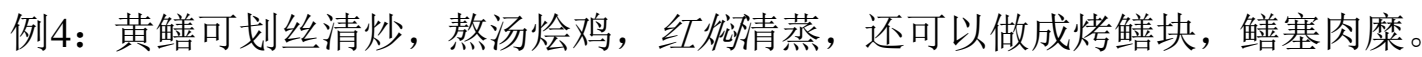

在翻译此句时, 由于缺少生活经验, 学生对 “红润”一词感到疑惑。后在教师的引导下, 通过网络寻找到了答案: 中式烹饪方法中, 红润是一种方法, 指的是烹制过程中使用酱油, 菜的色泽偏红。因此颜色词本身是不用翻译的，可以意译为braised in brown sauce。在此基础 上, 教师可以乘热打铁, 引导学生对饮食文化翻译中的常见词语进行查询、归纳和记忆, 从 而提高学生的语言外能力, 为今后遇见类似文本的翻译做好知识储备。

\section{4 利用网络, 训练工具能力}

当今时代已是网络时代，翻译与互联网技术的结合越来越紧密，基于互联网的工具能力 是翻译能力不可或缺的构成部分, 译者必须熟悉主流的翻译技术和翻译工具来提高翻译效率, 产出高质量的译文。根据韩孟奇的研究, 网络词典、搜索引擎、语料库、平行文本、网站论 坛等网络工具能有效地提高汉译英的表达能力。[12]

笔者曾让学生翻译过学校大学生心理健康指导中心的 “来访者须知”。因为对此类文本 较为陌生, 学生感觉无从下手, 翻译后的文本自己感觉也不满意。此时可以告诉学生, 心理 辅导在国外高校也很普遍, 只要查找相关的平行文本, 应该有不少可资利用的通行表达。首 先要确定心理健康指导的英文名, 然后在谷歌中输入 “psychological counseling site:edu”, 限 制搜索对象为国外高校网页中含有“psychological counseling”的内容, 就可以找到对应的平行 文本。在阅读了数篇平行文本后, 不难发现, 国外高校的心理咨询服务有其固有的行文方式 和特点, 往往将保密性(confidentiality)放在首位, 然后涉及宗旨(mission), 服务内容(services), 联系方式(contact information)等内容。通过这种方式, 学生掌握了如何查阅平行文本, 并且还 能通过比读意识到要对原文语篇进行重组改写, 进而掌握restructuring这一翻译策略。 


\section{5. 结束语}

汉英应用翻译翻译能力是由双语交际能力、翻译专业知识、策略能力、语言外能力和工 具能力五位一体构成的总和。发展学习者的翻译能力是翻译课的主要任务。汉英应用翻译是 译出翻译活动, 双语交际能力是核心能力, 而译者的翻译专业知识、策略能力越强, 译文的 可读性越高, 翻译腔越少; 译者的语言外能力和工具能力越高, 工作效率和准确度就越高。

\section{致谢}

本文受福建农林大学2015年第二批本科教育教学改革研究一般项目《本科生应用翻译能 力培养研究》(111415243), 外语教学与研究出版社有限责任公司大学外语教学科研项目《高 校对外宣介的翻译伦理研究》(20150016)及2016年福建省中青年教师教育科研项目《外宣翻 译的译者责任伦理研究》(JAS160166)资助。

\section{References}

[1] Information on http://download.china.cn/en/pdf/Report2014.pdf.

[2] H. Ma: A Study of Translation Competence from Chinese into English (Beijing Normal University Publishing Group, China 2013), p. 42, 70.

[3] S. Wang: On Translation Teaching (Shanghai Foreign Language Education Press, China 2013), pp.27-35.

[4] PACTE, Results of the validation of the PACTE translation competence model: acceptability and decision making, Across Languages and Cultures, vol.10, pp. 207-230, 2009.

[5] Y. Wu, Translation competence and translator training for Chinese culture "Going Out", Foreign Language Research, vol.1, pp. 148-153, 2015.

[6] H. Lin: A Companion for Chinese Translators (Hubei Education Press, China 2005), p. 179.

[7] H. Wang, A study on the components and layers of development C-E translation competence, Foreign Languages Research, vol. 2, pp. 72-76, 2012.

[8] H. Ma, Developing Learners' C-E translation competence, Chinese Translators Journal, vol. 31, pp. 39-44, 2010.

[9] P. Newmark: Approaches to Translation (Pergamon Press Ltd., England 1981). Oxford: 1981

[10]K. Lin, Adapt-Borrow-Create: developing pragmatic translation theories, Shanghai Journal of Translators, vol. 3, pp. 5-8, 2007.

[11]C. Schaffner: Developing Translation Competence (John Benjamins Publishing Company, The Netherlands 2000).

[12]M. Han, The development of language proficiency in C-E translation with internet technology, Technology Enhanced Foreign Language Education, vol. 6, pp. 42-46, 2015. 